PROCEEDINGS OF THE

AMERICAN MATHEMATICAL SOCIETY

Volume 129, Number 10, Pages 2931-2940

S 0002-9939(01)05893-2

Article electronically published on February 15, 2001

\title{
ROUGH SINGULAR INTEGRALS ASSOCIATED TO SURFACES OF REVOLUTION
}

\author{
SHANZHEN LU, YIBIAO PAN, AND DACHUN YANG \\ (Communicated by Christopher D. Sogge)
}

\begin{abstract}
Let $1<p<\infty$ and $n \geq 2$. The authors establish the $L^{p}\left(\mathbb{R}^{n+1}\right)$ boundedness for a class of singular integral operators associated to surfaces of revolution, $\left\{(t, \phi(|t|)): t \in \mathbb{R}^{n}\right\}$, with rough kernels, provided that the corresponding maximal function along the plane curve $\{(t, \phi(|t|)): t \in \mathbb{R}\}$ is bounded on $L^{p}\left(\mathbb{R}^{2}\right)$.
\end{abstract}

\section{INTRODUCTION}

Let $n \geq 2$ and $y \in \mathbb{R}^{n}$. For the Calderón-Zygmund type kernel

$$
K(y)=\frac{\Omega(y)}{|y|^{n}} b(|y|)
$$

and a suitable function $\phi$ on $[0, \infty)$, we define the singular integral operator $T$ along the surface

$$
\Gamma=\left\{(y, \phi(|y|)): y \in \mathbb{R}^{n}\right\}
$$

by

$$
T f(x, s)=\mathrm{p} \cdot \mathrm{v} \cdot \int_{\mathbb{R}^{n}} f(x-y, s-\phi(|y|)) K(y) d y .
$$

Here and in what follows, we always assume that $b$ is a measurable function on $[0, \infty), \Omega$ is homogeneous of order zero on $\mathbb{R}^{n}$, integrable on $S^{n-1}$ and satisfies

$$
\int_{S^{n-1}} \Omega(y) d \sigma(y)=0 .
$$

The kernel $K(y)$, which has radial roughness introduced by the factor $b(|y|)$, was first studied by R. Fefferman in the context of singular integrals on $\mathbb{R}^{n}([9])$.

In [10], Kim, Wainger, Wright and Ziesler proved the following theorem.

Received by the editors November 22, 1999 and, in revised form, February 10, 2000.

1991 Mathematics Subject Classification. Primary 42B20; Secondary 42B25, 47B38, 42B30, 43A90.

Key words and phrases. Curve, surface of revolution, singular integral, maximal operator, rough kernel, Hardy space, sphere.

The first author was supported by the NNSF of China.

The second author was supported by the NNSF of China.

The third author was supported by the Croucher Foundation Chinese Visitorships 1999-2000 of Hong Kong and the NNSF of China. 
Theorem A $([10])$. Let $\phi \in C^{2}([0, \infty))$ be convex, increasing and $\phi(0)=0$. Let $\Omega \in C^{\infty}\left(S^{n-1}\right)$ satisfy (2) and $b \equiv 1$. Then $T$ in (1) is bounded on $L^{p}\left(\mathbb{R}^{n+1}\right)$ for $1<p<\infty$.

In 3, Chen and Fan generalized the above result by requiring that $\Omega$ belongs to a Block space introduced in [11] and $b \in L^{\infty}([0, \infty))$.

Theorem B ([3]). Suppose $\Omega \in B_{r}^{\beta}\left(S^{n-1}\right)$ for some $\beta>0$ and $r>1$. If the maximal operator $\nu_{\phi}$ on $\mathbb{R}$ given by

$$
\left(\nu_{\phi} g\right)(x)=\sup _{k \in \mathbb{Z}} \frac{1}{2^{k}} \int_{2^{k}}^{2^{k+1}}|g(x-\phi(t))| d t
$$

is a bounded operator on $L^{p}(\mathbb{R})$ for $1<p<\infty$, then $T$ is bounded on $L^{p}\left(\mathbb{R}^{n+1}\right)$ for $1<p<\infty$.

The main purpose of this paper is to consider the $L^{p}$ boundedness of $T$ when $\Omega \in H^{1}\left(S^{n-1}\right)$, the Hardy space on the sphere; see [5] and 4] for the definition. The method that we use in this paper comes from the work of Duoandikoetxea and Rubio de Francia ([6]) and its extension obtained in Fan-Pan ([8]).

To state our main result, we need to introduce the maximal function $\mathcal{M}_{\phi}$ associated to the plane curve $\{(x, \phi(|x|)): x \in \mathbb{R}\}$. For any measurable function $f$ on $\mathbb{R}^{2}, \mathcal{M}_{\phi} f$ is defined by

$$
\mathcal{M}_{\phi} f\left(x_{1}, x_{2}\right)=\sup _{k \in \mathbb{Z}} \frac{1}{2^{k}} \int_{2^{k}}^{2^{k+1}}\left|f\left(x_{1}-t, x_{2}-\phi(|t|)\right)\right| d t .
$$

Here is our main theorem.

Theorem 1. Let $\phi:[0, \infty) \rightarrow \mathbb{R}$ be continuously differentiable on $(0, \infty)$ and satisfy

$$
|\phi(t)-\phi(0)| \leq C t^{\alpha}
$$

for some $\alpha>0$ and small $t$, where $C$ is a constant independent of $t$. Let $\Omega \in$ $H^{1}\left(S^{n-1}\right), b \in L^{\infty}([0, \infty))$ and $T$ be given by $(1)$. Then $T$ is bounded on $L^{p}\left(\mathbb{R}^{n+1}\right)$ for $1<p<\infty$, provided that $\mathcal{M}_{\phi}$ in (3) is bounded on $L^{p}\left(\mathbb{R}^{2}\right)$.

The condition imposed on $\phi(t)$ for $t \sim 0$ ensures that the integral in (1) exists in principle-value sense when, say, $f \in \mathcal{S}\left(\mathbb{R}^{n+1}\right)$.

The $L^{p}\left(\mathbb{R}^{2}\right)$ boundedness of $\mathcal{M}_{\phi}$ is known for many $\phi$ 's. Below we shall mention a few prominent cases:

(i) If $\phi$ is a real-valued polynomial, then $\mathcal{M}_{\phi}$ is bounded on $L^{p}\left(\mathbb{R}^{2}\right)$ for $p>1$; see [13.

(ii) Let $h(t)=t \phi^{\prime}(t)-\phi(t)$ for $t>0$. If $\phi: \mathbb{R} \rightarrow \mathbb{R}$ is of class $C^{2}(0, \infty)$, convex on $[0, \infty)$ and $\phi(0)=\phi^{\prime}(0)=0$ and there exists an $\varepsilon>0$ so that for each $t>0$, $h^{\prime}(t)>\varepsilon h(t) / t$, then $\mathcal{M}_{\phi}$ in (3) is bounded on $L^{p}\left(\mathbb{R}^{2}\right)$ for $p>1$; see Theorem 1.5 in [2]. Moreover, if $\phi$ is either even or odd, convex on $[0, \infty)$, and there exists a $0<C<\infty$ so that for each $t>0, \phi^{\prime}(C t) \geq 2 \phi^{\prime}(t)$, then $\mathcal{M}_{\phi}$ in (3) is bounded on $L^{p}\left(\mathbb{R}^{2}\right)$ for $p>1$. For details, see [1] or [2].

(iii) For $\phi(t)=t^{\alpha}$ with $\alpha \in(0,1], \mathcal{M}_{\phi}$ is bounded on $L^{p}\left(\mathbb{R}^{2}\right)$ for $p>1$; see [12]. 


\section{Proof of Theorem 1}

We begin with the definition of the space $H^{1}\left(S^{n-1}\right)$. For $f \in L^{1}\left(S^{n-1}\right)$ and $x \in S^{n-1}$, we define

$$
P^{+} f(x)=\sup _{0<t<1}\left|\int_{S^{n-1}} P_{t x}(y) f(y) d \sigma(y)\right|,
$$

where

$$
P_{t x}(y)=\frac{1-t^{2}}{|y-t x|^{n}}
$$

for $y \in S^{n-1}$.

Definition 1. An integrable function $f$ on $S^{n-1}$ is in the space $H^{1}\left(S^{n-1}\right)$ if and only if

$$
\left\|P^{+} f\right\|_{L^{1}\left(S^{n-1}\right)}=\int_{S^{n-1}}\left|P^{+} f(x)\right| d \sigma(x)<\infty
$$

and we define

$$
\|f\|_{H^{1}\left(S^{n-1}\right)}=\left\|P^{+} f\right\|_{L^{1}\left(S^{n-1}\right)} .
$$

A very useful characterization of the space $H^{1}\left(S^{n-1}\right)$ is its atomic decomposition. Let us first recall the definition of atoms.

Definition 2. A function $a(\cdot)$ on $S^{n-1}$ is a regular atom if there exist $\xi \in S^{n-1}$ and $\rho \in(0,2]$ such that

(i) $\operatorname{supp} a \subset S^{n-1} \cap B(\xi, \rho)$, where $B(\xi, \rho)=\left\{y \in \mathbb{R}^{n}:|y-\xi|<\rho\right\}$;

(ii) $\|a\|_{L^{\infty}\left(S^{n-1}\right)} \leq \rho^{-n+1}$;

(iii) $\int_{S^{n-1}} a(y) d \sigma(y)=0$.

A function $a(\cdot)$ on $S^{n-1}$ is an exceptional atom if $a(\cdot) \in L^{\infty}\left(S^{n-1}\right)$ and

$$
\|a\|_{L^{\infty}\left(S^{n-1}\right)} \leq 1 .
$$

The following can be found in [5] and [4].

Lemma 1. For any $f \in H^{1}\left(S^{n-1}\right)$ there are complex numbers $\lambda_{j}$ and atoms (regular or exceptional) $a_{j}$ such that

$$
f=\sum_{j} \lambda_{j} a_{j}
$$

and

$$
\|f\|_{H^{1}\left(S^{n-1}\right)} \sim \sum_{j}\left|\lambda_{j}\right|
$$

The following lemma is a simple corollary of Theorem B.

Lemma 2. Let $\phi$ be the same as in Theorem 1. Let $\Omega \in L^{r}\left(S^{n-1}\right)$ for some $1<r \leq \infty, n \geq 2$ and $T$ be given by (1). Then $T$ is bounded on $L^{p}\left(\mathbb{R}^{n+1}\right)$ for $1<p<\infty$, provided that $\mathcal{M}_{\phi}$ in (3) is bounded on $L^{p}\left(\mathbb{R}^{2}\right)$. 
Proof. It suffices to show that the $L^{p}\left(\mathbb{R}^{2}\right)$ boundedess of $\mathcal{M}_{\phi}$ implies the $L^{p}(\mathbb{R})$ boundedess of the maximal operator $\nu_{\phi}$.

For $N \in \mathbb{N}$, let

$$
\left(\nu_{\phi}^{N} g\right)(x)=\sup _{-\infty<k \leq N} \frac{1}{2^{k}} \int_{2^{k}}^{2^{k+1}}|g(x-\phi(t))| d t .
$$

Then for $f(x, y)=\chi_{\left[0,2^{N+2}\right]}(x) g(y)$,

$$
\chi_{\left[0,2^{N+1}\right]}(x)\left(\nu_{\phi}^{N} g\right)(y) \leq\left(\mathcal{M}_{\phi} f\right)(x, y) .
$$

Thus

$$
2^{(N+1) / p}\left\|\nu_{\phi}^{N} g\right\|_{L^{p}(\mathbb{R})} \leq\left\|\mathcal{M}_{\phi} f\right\|_{L^{p}\left(\mathbb{R}^{2}\right)} \leq C_{p}\|f\|_{L^{p}\left(\mathbb{R}^{2}\right)}=C_{p} 2^{(N+2) / p}\|g\|_{L^{p}(\mathbb{R})} .
$$

By letting $\mathbb{N} \rightarrow \infty$ (after dividing both sides by $2^{(N+1) / p}$ ), we obtain

$$
\left\|\nu_{\phi} g\right\|_{L^{p}(\mathbb{R})} \leq C_{p} 2^{1 / p}\|g\|_{L^{p}(\mathbb{R})} .
$$

This finishes the proof of Lemma 2 .

The following lemma in [7] is one of our main tools.

Lemma 3. Let $l, m \in \mathbb{N}$ and $\left\{\sigma_{s, k}: 0 \leq s \leq l\right.$ and $\left.k \in \mathbb{Z}\right\}$ be a family of measures on $\mathbb{R}^{m}$ with $\sigma_{0, k}=0$ for every $k \in \mathbb{Z}$. Let $\left\{\alpha_{s j}: 1 \leq s \leq l\right.$ and $1 \leq$ $j \leq 2\} \subset(0, \infty),\left\{\eta_{s}: 1 \leq s \leq l\right\} \subset(0, \infty) \backslash\{1\},\left\{M_{s}: 1 \leq s \leq l\right\} \subset \mathbb{N}$, and $L_{s}: \mathbb{R}^{m} \rightarrow \mathbb{R}^{M_{s}}$ be linear transformations for $1 \leq s \leq l$. Suppose

(i) $\left\|\sigma_{s, k}\right\| \leq 1$ for $k \in \mathbb{Z}$ and $1 \leq s \leq l$;

(ii) $\left|\widehat{\sigma}_{s, k}(\xi)\right| \leq C\left(\eta_{s}^{k}\left|L_{s} \xi\right|\right)^{-\alpha_{s 2}}$ for $\xi \in \mathbb{R}^{m}, k \in \mathbb{Z}$ and $1 \leq s \leq l$;

(iii) $\left|\widehat{\sigma}_{s, k}-\widehat{\sigma}_{s-1, k}(\xi)\right| \leq C\left(\eta_{s}^{k}\left|L_{s} \xi\right|\right)^{\alpha_{s 1}}$ for $\xi \in \mathbb{R}^{m}, k \in \mathbb{Z}$ and $1 \leq s \leq l$;

(iv) For some $q>1$, there exists $A_{q}>0$ such that

$$
\left\|\sup _{k \in \mathbb{Z}}|| \sigma_{s, k}|* f|\right\|_{L^{q}\left(\mathbb{R}^{m}\right)} \leq A_{q}\|f\|_{L^{q}\left(\mathbb{R}^{m}\right)}
$$

for all $f \in L^{q}\left(\mathbb{R}^{m}\right)$ and $1 \leq s \leq l$.

Then for every $p \in\left(\frac{2 q}{q+1}, \frac{2 q}{q-1}\right)$, there exists a positive constant $C_{p}$ such that

$$
\left\|\sum_{k \in \mathbb{Z}} \sigma_{l, k} * f\right\|_{L^{p}\left(\mathbb{R}^{m}\right)} \leq C_{p}\|f\|_{L^{p}\left(\mathbb{R}^{m}\right)}
$$

and

$$
\left\|\left(\sum_{k \in \mathbb{Z}}\left|\sigma_{l, k} * f\right|^{2}\right)^{1 / 2}\right\|_{L^{p}\left(\mathbb{R}^{m}\right)} \leq C_{p}\|f\|_{L^{p}\left(\mathbb{R}^{m}\right)}
$$

hold for all $f \in L^{p}\left(\mathbb{R}^{m}\right)$. The constant $C_{p}$ is independent of the linear transformations $\left\{L_{s}\right\}_{s=1}^{l}$.

The following result is just Lemma 5.1 in 7 , which follows immediately from Lemma 6.2 in [8] and is an extension of an earlier theorem due to Duoandikoetxea and Rubio de Francia in [6].

Lemma 4. Let $s, m \in \mathbb{N}, \eta \in(0, \infty) \backslash\{1\}, \delta_{1}, \delta_{2}>0$, and $L: \mathbb{R}^{s} \rightarrow \mathbb{R}^{m}$ be a linear transformation. Suppose that $\left\{\sigma_{k}\right\}_{k \in \mathbb{Z}}$ is a sequence of measures on $\mathbb{R}^{m}$ 
satisfying:

(i) $\left\|\sigma_{k}\right\| \leq 1$ for $k \in \mathbb{Z}$;

(ii) $\left|\widehat{\sigma_{k}}(\xi)\right| \leq C\left[\min \left\{\left(\eta^{k}|L \xi|\right)^{\delta_{1}},\left(\eta^{k}|L \xi|\right)^{-\delta_{2}}\right\}\right]$ for $\xi \in \mathbb{R}^{s}$ and $k \in \mathbb{Z}$;

(iii) For some $q>1$, there exists $A_{q}>0$ such that

$$
\left\|\sigma^{*}(f)\right\|_{L^{q}\left(\mathbb{R}^{m}\right)}=\left\|\sup _{k \in \mathbb{Z}}|| \sigma_{k}|* f|\right\|_{L^{q}\left(\mathbb{R}^{m}\right)} \leq A_{q}\|f\|_{L^{q}\left(\mathbb{R}^{m}\right)}
$$

for all $f \in L^{q}\left(\mathbb{R}^{m}\right)$.

Then for $p \in\left(\frac{2 q}{q+1}, \frac{2 q}{q-1}\right)$, there exists a positive constant $C_{p}=C\left(p, s, m, \eta, \delta_{1}, \delta_{2}\right)$ such that

$$
\left\|\sum_{k \in \mathbb{Z}} \sigma_{k} * f\right\|_{L^{p}\left(\mathbb{R}^{m}\right)} \leq C_{p}\|f\|_{L^{p}\left(\mathbb{R}^{m}\right)}
$$

and

$$
\left\|\left(\sum_{k \in \mathbb{Z}}\left|\sigma_{k} * f\right|^{2}\right)^{1 / 2}\right\|_{L^{p}\left(\mathbb{R}^{m}\right)} \leq C_{p}\|f\|_{L^{p}\left(\mathbb{R}^{m}\right)}
$$

hold for all $f \in L^{p}\left(\mathbb{R}^{m}\right)$. The constant $C_{p}$ is independent of the linear transformation $L$.

In order to handle truncation in the phase space, we need the following useful lemma, which is Lemma 6.4 in 8 .

Lemma 5. For $s \leq d$, let $H: \mathbb{R}^{s} \rightarrow \mathbb{R}^{s}$ and $G: \mathbb{R}^{d} \rightarrow \mathbb{R}^{d}$ be two nonsingular linear transformations and $\varphi \in \mathcal{S}\left(\mathbb{R}^{s}\right)$. Define $J$ and $X_{r}=X_{r}(\varphi, G, H)$ by

$$
(J f)(x)=f\left(G^{t}\left(H^{t} \otimes i d_{\mathbb{R}^{d-s}}\right)\right)(x)
$$

and

$$
X_{r} f(x)=J^{-1}\left(\left(\left|\Phi_{r}\right| \otimes \delta_{\mathbb{R}^{d-s}}\right) * J f\right)(x),
$$

where $x \in \mathbb{R}^{d}, r>0, G^{t}$ and $H^{t}$ are respectively the transposes of $G$ and $H, i d_{\mathbb{R}^{d-s}}$ is the identity operator on $\mathbb{R}^{d-s}, \delta_{\mathbb{R}^{d-s}}$ is the Dirac delta operator on $\mathbb{R}^{d-s}$, and $\Phi \in \mathcal{S}\left(\mathbb{R}^{s}\right)$ satisfies $\widehat{\Phi}=\varphi$. Let $X=X(\varphi, G, H)$ be given by

$$
X f(x)=\sup _{r>0}\left|X_{r} f(x)\right| .
$$

Then for $1<p \leq \infty$, there exists a positive constant $C_{p}=C(p, \varphi, s, d)$ such that

$$
\|X f\|_{L^{p}\left(\mathbb{R}^{d}\right)} \leq C_{p}\|f\|_{L^{p}\left(\mathbb{R}^{d}\right)}
$$

for all $f \in L^{p}\left(\mathbb{R}^{d}\right)$. The constant $C_{p}$ is independent of the linear transformations $G$ and $H$.

Now let $\Delta_{\gamma}(0, \infty)$ denote the set of functions $b$ on $(0, \infty)$ satisfying

$$
\sup _{R>0} \frac{1}{R} \int_{0}^{R}|b(t)|^{\gamma} d t<\infty .
$$

For $y=\left(y_{1}, \cdots, y_{n}\right) \in \mathbb{R}^{n}$, let $\widetilde{y}=\left(y_{1}, \cdots, y_{n-1}\right) \in \mathbb{R}^{n-1}$. We denote the north pole $(0, \cdots, 0,1)$ on $S^{n-1}$ by $\rho_{1}$. Let $F: \mathbb{R} \times \mathbb{R}^{n} \rightarrow \mathbb{R}$ be of the form

$$
F(t, y)=t^{l} q(\widetilde{y})+W_{1}(t, y)+W_{2}(t),
$$


where $q: \mathbb{R}^{n-1} \rightarrow \mathbb{R}$ is a polynomial, $W_{1}$ satisfies

$$
\frac{\partial^{l} W_{1}}{\partial t^{l}}(t, y) \equiv 0
$$

and $W_{2}(\cdot)$ is an arbitrary function.

The following estimate on the oscillatory integrals is Proposition 5.3 in [8] and is of considerable importance to us.

Lemma 6. Let $\rho \in(0,1 / 4), l \in \mathbb{N}, m \geq 0, q(\widetilde{y})=\sum_{j=0}^{m} q_{j}(\widetilde{y})$, where $q_{j}(\cdot)$ is a homogeneous polynomial of degree $j$ on $\mathbb{R}^{n-1}$ for $0 \leq j \leq m$. Let $F(t, y)$ be given by (4) and (5). Suppose that $b \in \Delta_{\gamma}$ for some $\gamma>1$ and $\Omega(\cdot)$ is a function satisfying

(a) $\operatorname{supp}(\Omega) \subset B\left(\rho_{1}, \rho\right)$;

(b) $\|\Omega\|_{L^{\infty}\left(S^{n-1}\right)} \leq \rho^{-n+1}$.

If we assume $q_{m}(\widetilde{y})=\sum_{|\beta|=m} \alpha_{\beta} \widetilde{y}^{\beta}$ and $\left\|q_{m}\right\|=\sum_{|\beta|=m}\left|\alpha_{\beta}\right|$, then there exists a positive constant $C$ such that

$$
\int_{2^{k}}^{2^{k+1}}\left|\int_{S^{n-1}} e^{i F(t, y)} \Omega(y) d \sigma(y)\right| \frac{|b(t)|}{t} d t \leq C\left(2^{k l} \rho^{m}\left\|q_{m}\right\|\right)^{-\frac{1}{4 m l \gamma}} .
$$

The constant $C$ may depend on $l, m, n$, and $b(\cdot)$, but it is independent of $k, \rho$, $W_{1}(\cdot, \cdot), W_{2}(\cdot)$, and the coefficients of $q(\cdot)$.

Proof of Theorem 1. Since $\Omega \in H^{1}\left(S^{n-1}\right)$ and $\int_{S^{n-1}} \Omega(y) d \sigma(y)=0$, there are regular atoms $a_{j}(\cdot)$ and $\left\{C_{j}\right\} \subset \mathbb{C}$ such that

$$
\Omega(y)=\sum_{j} C_{j} a_{j}(y)
$$

by Lemma 1.

Therefore, we only need to be concerned with the case where $\Omega(y)$ is a regular atom on $S^{n-1}$. By Lemma 2 and using a rotation if necessary, we may assume that there is a $\rho \in(0,1 / 4)$ such that

$$
\begin{aligned}
& \operatorname{supp}(\Omega) \subset B\left(\rho_{1}, \rho\right), \text { where } \rho_{1}=(0, \cdots, 0,1) ; \\
& \|\Omega\|_{L^{\infty}\left(S^{n-1}\right)} \leq \rho^{-(n-1)} ; \int_{S^{n-1}} \Omega(y) d \sigma(y)=0 .
\end{aligned}
$$

For any integrable function $a(\cdot)$ on $S^{n-1}$ and a suitable mapping $\Gamma: \mathbb{R}^{n} \rightarrow \mathbb{R}^{n+1}$, we define the sequence of measures $\left\{\sigma_{a, \Gamma, k}\right\}_{k \in \mathbb{Z}}$ by

$$
\int_{\mathbb{R}^{n+1}} F d \sigma_{a, \Gamma, k}=\int_{\left\{y \in \mathbb{R}^{n}: 2^{k} \leq|y|<2^{k+1}\right\}} F(\Gamma(y)) \frac{a(y)}{|y|^{n}} b(|y|) d y .
$$

For $y \in \mathbb{R}^{n} \backslash\{0\}$, let $\widetilde{y}=\left(y_{1} /|y|, \cdots, y_{n-1} /|y|\right)$. Let $N=\left[\frac{3(n-1)}{2}\right]+2$ (this $N$ is chosen so that we can have both (9) and (10) for $j=N)$. For $j=1, \cdots, N-2$, let $b_{j}=(-1)^{j} \frac{1}{2}\left(\frac{1}{2}-1\right) \cdots\left(\frac{1}{2}-j+1\right) / j !$. Thus

$$
\left|(1-t)^{1 / 2}-1-\sum_{l=1}^{j-1} b_{l} t^{l}\right| \leq C_{j} t^{j}
$$

for $t \in[0,1 / 4]$. 
We now define the mappings $\Gamma_{0}, \Gamma_{1}, \cdots, \Gamma_{N}$ by

$\Gamma_{N}(y)=(y, \phi(|y|))$,

$\Gamma_{j}(y)=\left(|y| \widetilde{y},|y|\left(1+b_{1}|\widetilde{y}|^{2}+\cdots+b_{j-1}|\widetilde{y}|^{2(j-1)}\right), \phi(|y|)\right), j=2, \cdots, N-1$,

$\Gamma_{1}(y)=(|y| \widetilde{y},|y|, \phi(|y|))$,

and

$$
\Gamma_{0}(y)=(0,|y|, \phi(|y|))
$$

For $\xi=\left(\xi_{1}, \ldots, \xi_{n}\right) \in \mathbb{R}^{n}$ and $\eta \in \mathbb{R}$, we shall establish estimates (ii) and (iii) in Lemma 3 for $\left\{\left|\widehat{\sigma}_{\Omega, \Gamma_{j}, k}(\xi, \eta)\right|: 1 \leq j \leq N\right.$ and $\left.k \in \mathbb{Z}\right\}$. By an inequality on page 551 of [6], we have

$$
\begin{aligned}
\left|\widehat{\sigma}_{\Omega, \Gamma_{N}, k}(\xi, \eta)\right| & \leq \int_{2^{k}}^{2^{k+1}}\left|\int_{S^{n-1}} e^{-i[t \xi \cdot y+\eta \phi(t)]} \Omega(y) d \sigma(y)\right||b(t)| \frac{d t}{t} \\
& \leq C\left[2^{k}|\xi|\right]^{-1 / 6}\|\Omega\|_{L^{2}\left(S^{n-1}\right)} \\
& \leq C\left[2^{k}\left|\rho^{3(n-1)} \xi_{n}\right|\right]^{-1 / 6} .
\end{aligned}
$$

One observes that the variable $\eta$ does not appear in the previous inequality. The same is true for the Fourier estimates obtained from here on.

Now, for $2 \leq j \leq N-1$, we have

$$
\left|\widehat{\sigma}_{\Omega, \Gamma_{j}, k}(\xi, \eta)\right| \leq \int_{2^{k}}^{2^{k+1}}\left|\int_{S^{n-1}} e^{-i t\left[\left(\xi_{1}, \cdots, \xi_{n-1}\right) \cdot \widetilde{y}+\xi_{n} \sum_{s=1}^{j-1} b_{s}|\widetilde{y}|^{2 s}\right]} \Omega(y) d \sigma(y)\right||b(t)| \frac{d t}{t} .
$$

By applying Lemma 6 with $q(\widetilde{y})=-\left[\left(\xi_{1}, \cdots, \xi_{n-1}\right) \cdot \widetilde{y}+\xi_{n} \sum_{s=1}^{j-1} b_{s}|\widetilde{y}|^{2 s}\right], m=$ $2(j-1), \gamma=2$ and $l=1$, we obtain

$$
\left|\widehat{\sigma}_{\Omega, \Gamma_{j}, k}(\xi, \eta)\right| \leq C\left[2^{k}\left|\rho^{2(j-1)} \xi_{n}\right|\right]^{-\frac{1}{16(j-1)}} .
$$

Finally, by Lemma 6 with $m=1, \gamma=2$ and $l=1$, we have

$$
\begin{aligned}
\left|\widehat{\sigma}_{\Omega, \Gamma_{1}, k}(\xi, \eta)\right| & \leq \int_{2^{k}}^{2^{k+1}}\left|\int_{S^{n-1}} e^{-i t\left[\left(\xi_{1}, \cdots, \xi_{n-1}\right) \cdot \widetilde{y}\right]} \Omega(y) d \sigma(y)\right||b(t)| \frac{d t}{t} \\
& \leq C\left[2^{k}\left|\rho\left(\xi_{1}, \cdots, \xi_{n-1}\right)\right|\right]^{-\frac{1}{8}} .
\end{aligned}
$$

Let

$$
\begin{aligned}
& L_{1}(\xi, \eta)=\rho\left(\xi_{1}, \cdots, \xi_{n-1}\right), \theta_{1}=\frac{1}{8} \\
& L_{j}(\xi, \eta)=\rho^{2(j-1)} \xi_{n}, \theta_{j}=\frac{1}{16(j-1)}, 2 \leq j \leq N-1 ; \\
& L_{N}(\xi, \eta)=\rho^{3(n-1)} \xi_{n}, \theta_{N}=\frac{1}{6} .
\end{aligned}
$$

Then by (6)-(8), we have

$$
\left|\widehat{\sigma}_{\Omega, \Gamma_{j}, k}(\xi, \eta)\right| \leq C\left[2^{k}\left|L_{j}(\xi, \eta)\right|\right]^{-\theta_{j}}
$$

for $1 \leq j \leq N, k \in \mathbb{Z}, \xi \in \mathbb{R}^{n}$ and $\eta \in \mathbb{R}$. Next we shall verify that for $(\xi, \eta) \in$ $\mathbb{R}^{n+1}, k \in \mathbb{Z}$ and $1 \leq j \leq N$,

$$
\left|\widehat{\sigma}_{\Omega, \Gamma_{j}, k}(\xi, \eta)-\widehat{\sigma}_{\Omega, \Gamma_{j-1}, k}(\xi, \eta)\right| \leq C 2^{k}\left|L_{j}(\xi, \eta)\right| .
$$


Let us begin with $j=N$. In this case, we have

$$
\begin{aligned}
& \left|\widehat{\sigma}_{\Omega, \Gamma_{N}, k}(\xi, \eta)-\widehat{\sigma}_{\Omega, \Gamma_{N-1}, k}(\xi, \eta)\right| \\
& \quad \leq \int_{2^{k}}^{2^{k+1}}\left|\int_{S^{n-1}}\left(e^{-i t \xi_{n}\left[\left(1-|\widetilde{y}|^{2}\right)^{1 / 2}-1-\sum_{s=1}^{N-2} b_{s}|\widetilde{y}|^{2 s}\right]}-1\right) \Omega(y) d \sigma(y)\right||b(t)| \frac{d t}{t} \\
& \quad \leq C \int_{2^{k}}^{2^{k+1}} t\left|\xi_{n}\right| \rho^{2(N-1)}\|\Omega\|_{L^{1}\left(S^{n-1}\right)} \frac{d t}{t} \\
& \quad \leq C 2^{k}\left|\rho^{3(n-1)} \xi_{n}\right|=C\left|2^{k} L_{N}(\xi, \eta)\right| .
\end{aligned}
$$

For $2 \leq j \leq N-1$, we have

$$
\begin{aligned}
& \left|\widehat{\sigma}_{\Omega, \Gamma_{j}, k}(\xi, \eta)-\widehat{\sigma}_{\Omega, \Gamma_{j-1}, k}(\xi, \eta)\right| \\
& \quad \leq \int_{2^{k}}^{2^{k+1}} \int_{S^{n-1}}\left|e^{-i t \xi_{n} b_{j-1}|\widetilde{y}|^{2(j-1)}}-1\right||\Omega(y)| d \sigma(y)|b(t)| \frac{d t}{t} \\
& \quad \leq C 2^{k}\left|\rho^{2(j-1)} \xi_{n}\right|=C\left|2^{k} L_{j}(\xi, \eta)\right| .
\end{aligned}
$$

Finally, for $j=1$, we have

$$
\begin{aligned}
& \left|\widehat{\sigma}_{\Omega, \Gamma_{1}, k}(\xi, \eta)-\widehat{\sigma}_{\Omega, \Gamma_{0}, k}(\xi, \eta)\right| \\
& \quad \leq \int_{2^{k}}^{2^{k+1}} \int_{S^{n-1}}\left|e^{-i t\left(\xi_{1}, \cdots, \xi_{n-1}\right) \cdot \widetilde{y}}-1\right||\Omega(y)| d \sigma(y)|b(t)| \frac{d t}{t} \\
& \quad \leq C 2^{k} \rho\left|\left(\xi_{1}, \cdots, \xi_{n-1}\right)\right|=C\left|2^{k} L_{1}(\xi, \eta)\right| .
\end{aligned}
$$

This completes the proof of (10).

We still need to verify condition (iv) in Lemma 3 . It suffices to establish the $L^{p}\left(\mathbb{R}^{n}\right)$ boundedness of the operators $\sigma_{\Omega, j}^{*}$ defined by

$$
\sigma_{\Omega, j}^{*}(f)(x, s)=\sup _{k \in \mathbb{Z}}\left|\left(\sigma_{|\Omega|, \Gamma_{j}, k} * f\right)(x, s)\right|,
$$

where $j=1, \cdots, N, x \in \mathbb{R}^{n}, s \in \mathbb{R}$ and $1<p<\infty$.

Let us begin with $\sigma_{\Omega, 1}^{*}$ which is given by

$$
\sigma_{\Omega, 1}^{*}(f)(x, s)=\sup _{k \in \mathbb{Z}}\left|\left(\sigma_{|\Omega|, \Gamma_{1}, k} * f\right)(x, s)\right| .
$$

Choose $\theta \in C_{0}^{\infty}\left(\mathbb{R}^{n-1}\right)$ such that $\theta(t) \equiv 1$ for $|t| \leq 1 / 2$ and $\theta(t) \equiv 0$ for $|t| \geq 1$. For $k \in \mathbb{Z}$, we define $\nu_{k}$ by

$$
\widehat{\nu}_{k}(\xi, \eta)=\theta\left(2^{k} \rho\left(\xi_{1}, \cdots, \xi_{n-1}\right)\right) \widehat{\sigma}_{|\Omega|, \Gamma_{0}, k}(\xi, \eta)
$$

for $\xi \in \mathbb{R}^{n}$ and $\eta \in \mathbb{R}$. Let $\tau_{k}=\widehat{\sigma}_{|\Omega|, \Gamma_{1}, k}-\nu_{k}$. Then by $(10)$ and $\left|\widehat{\sigma}_{|\Omega|, \Gamma_{0}, k}(\xi, \eta)\right| \leq C$, we have

$$
\begin{aligned}
\left|\widehat{\tau}_{k}(\xi, \eta)\right| \leq & \left|\widehat{\sigma}_{|\Omega|, \Gamma_{1}, k}(\xi, \eta)-\widehat{\sigma}_{|\Omega|, \Gamma_{0}, k}(\xi, \eta)\right| \\
& +\left|1-\theta\left(2^{k} \rho\left(\xi_{1}, \cdots, \xi_{n-1}\right)\right)\right|\left|\widehat{\sigma}_{|\Omega|, \Gamma_{0}, k}(\xi, \eta)\right| \\
\leq & C\left[\left|2^{k} L_{1}(\xi, \eta)\right|+\left|2^{k} \rho\left(\xi_{1}, \cdots, \xi_{n-1}\right)\right|\right] \\
= & C 2^{k}\left|L_{1}(\xi, \eta)\right| .
\end{aligned}
$$

If $2^{k}\left|L_{1}(\xi, \eta)\right|>1$, by (9), we have

$$
\left|\widehat{\tau}_{k}(\xi, \eta)\right| \leq C\left(2^{k}\left|L_{1}(\xi, \eta)\right|\right)^{-1 / 8} .
$$


Thus,

$$
\left|\widehat{\tau}_{k}(\xi, \eta)\right| \leq C\left[\min \left\{2^{k}\left|L_{1}(\xi, \eta)\right|,\left(2^{k}\left|L_{1}(\xi, \eta)\right|\right)^{-1}\right\}\right]^{1 / 8}
$$

Let

$$
\tau^{*}(f)(x, s)=\sup _{k \in \mathbb{Z}}\left|\left(\left|\tau_{k}\right| * f\right)(x, s)\right|, \nu^{*}(f)(x, s)=\sup _{k \in \mathbb{Z}}\left|\left(\left|\nu_{k}\right| * f\right)(x, s)\right|
$$

and

$$
g_{\tau}(f)(x, s)=\left\{\sum_{k \in \mathbb{Z}}\left|\left(\tau_{k} * f\right)(x, s)\right|^{2}\right\}^{1 / 2} .
$$

Then

$$
\sigma_{|\Omega|, 1}^{*}(f)(x, s) \leq g_{\tau}(f)(x, s)+\nu^{*}(f)(x, s)
$$

and

$$
\begin{aligned}
\tau^{*}(f)(x, s) & \leq \sigma_{|\Omega|, 1}^{*}(|f|)(x, s)+\nu^{*}(|f|)(x, s) \\
& \leq g_{\tau}(|f|)(x, s)+2 \nu^{*}(|f|)(x, s) .
\end{aligned}
$$

By the $L^{p}\left(\mathbb{R}^{2}\right)$ boundedness of $\mathcal{M}_{\phi}$ and Lemma 5 , for $1<p<\infty$, we have

$$
\left\|\nu^{*}(|f|)\right\|_{L^{p}\left(\mathbb{R}^{n+1}\right)} \leq C_{p}\|f\|_{L^{p}\left(\mathbb{R}^{n+1}\right)} .
$$

Also, from (11), it is easy to deduce that

$$
\left\|g_{\tau}(f)\right\|_{L^{2}\left(\mathbb{R}^{n+1}\right)} \leq C\|f\|_{L^{2}\left(\mathbb{R}^{n+1}\right)} .
$$

Thus, (13) implies that

$$
\left\|\tau^{*}(f)\right\|_{L^{2}\left(\mathbb{R}^{n+1}\right)} \leq C\|f\|_{L^{2}\left(\mathbb{R}^{n+1}\right)} .
$$

By invoking Lemma 4, we obtain

$$
\left\|g_{\tau}(f)\right\|_{L^{p}\left(\mathbb{R}^{n+1}\right)} \leq C_{p}\|f\|_{L^{p}\left(\mathbb{R}^{n+1}\right)}
$$

for $4 / 3<p<4$. Thus, by (13) again, we obtain

$$
\left\|\tau^{*}(f)\right\|_{L^{p}\left(\mathbb{R}^{n+1}\right)} \leq C_{p}\|f\|_{L^{p}\left(\mathbb{R}^{n+1}\right)}
$$

for $4 / 3<p<4$. By using (14), (13) and repeating the preceding argument, we obtain

$$
\left\|g_{\tau}(f)\right\|_{L^{p}\left(\mathbb{R}^{n+1}\right)} \leq C_{p}\|f\|_{L^{p}\left(\mathbb{R}^{n+1}\right)}
$$

for $1<p<\infty$. Now, from (12), it follows that

$$
\left\|\sigma_{|\Omega|, 1}^{*}(f)\right\|_{L^{p}\left(\mathbb{R}^{n+1}\right)} \leq C_{p}\|f\|_{L^{p}\left(\mathbb{R}^{n+1}\right)}
$$

for $1<p<\infty$.

Similarly, we can show that

$$
\left\|\sigma_{|\Omega|, j}^{*}(f)\right\|_{L^{p}\left(\mathbb{R}^{n+1}\right)} \leq C_{p}\|f\|_{L^{p}\left(\mathbb{R}^{n+1}\right)}
$$

for $1 \leq j \leq N$. Now, by (9), (10), (15) and Lemma 3 , we have

$$
\left\|\sum_{k \in \mathbb{Z}} \sigma_{\Omega, \Gamma_{N}, k} * f\right\|_{L^{p}\left(\mathbb{R}^{n+1}\right)} \leq C_{p}\|f\|_{L^{p}\left(\mathbb{R}^{n+1}\right)}
$$


for $1<p<\infty$. Noting that

$$
\sum_{k \in \mathbb{Z}}\left(\sigma_{\Omega, \Gamma_{N}, k} * f\right)(x, s)=\int_{\mathbb{R}^{n}} f(x-y, s-\phi(|y|)) \frac{\Omega(y)}{|y|^{n}} b(|y|) d y,
$$

we thus obtain a proof of our theorem.

\section{ACKNOWLEDGEMENTS}

The authors would like to thank the referee for helpful comments.

\section{REFERENCES}

[1] Carlsson, H., Christ, M., Cordoba A., Duoandikoetxea, J. and Rubio de Francia, J. L., Vance, J., Wainger, S. and Weinberg, D., $L^{p}$ estimates for maximal functions and Hilbert transforms along flat convex plane curves in $\mathbb{R}^{2}$, Bull. Amer. Math. Soc. 14(1986), 263-267. MR 87f:42044

[2] Carbery, A., Christ, M., Vance, J., Wainger, S. and Watson, D. K., Operators associated to flat plane curves: $L^{p}$ estimates via dilation methods, Duke Math. J. 59(1989), 675-700. MR 91m: 42017

[3] Chen, L. and Fan, D., On singular integrals along surfaces related to block spaces, Integr. Equ. Oper. Theory 29(1997), 261-268. MR 99c:42027

[4] Coifman, R. and Weiss, G., Extension of Hardy spaces and their use in analysis, Bull. Amer. Math. Soc. 83(1977), 569-645. MR 56:6264

[5] Colzani, L., Hardy spaces on spheres, Ph.D. Thesis, Washington University, St Louis, 1982.

[6] Duoandikoetxea, J. and Rubio de Francia, J. L., Maximal and singular integral operators via Fourier transform estimates, Invent. Math. 84(1986), 541-561. MR 87f:42046

[7] Fan, D., Guo, K. and Pan, Y., $L^{p}$ estimates for singular integrals associated to homogeneous surfaces, Submitted.

[8] Fan, D. and Pan, Y., Singular integral operators with rough kernels supported by subvarieties, Amer. J. Math. 119(1997), 799-839. MR 99c:42029

[9] Fefferman, R., A note on singular integrals, Proc. Amer. Math. Soc. 74 (1979), 266-270. MR 81e: 42025

[10] Kim, W., Wainger, S., Wright, J. and Ziesler, S., Singular integrals and maximal functions associated to surfaces of revolution, Bull. London Math. Soc. 28(1996), 291-296. MR 97b:42029

[11] Taibleson, M. and Weiss, G., Spaces generated by Blocks, Publishing House of Beijing Normal Univ., Beijing, 1989. MR 87e:42008

[12] Ricci, F. and Stein, E. M., Multiparameter singular integrals and maximal functions, Ann. Inst. Fourier 42(1992), 637-670. MR 94d:42020

[13] Stein, E. M. and Wainger, S., Problems in harmonic analysis related to curvature, Bull. Amer. Math. Soc. 84(1978), 1239-1295. MR 80k:42023

Department of Mathematics, Beijing Normal University, Beijing 100875, The People's Republic of China

E-mail address: lusz@bnu.edu.cn

Department of Mathematics, City University of Hong Kong, Tat Chee Avenue, KowLOON, HONG KONG

E-mail address: yibiao+@pitt.edu

Department of Mathematics, Beijing Normal University, Beijing 100875, The People's Republic of China

E-mail address: dcyang@bnu.edu.cn 